\title{
Pricing a European Option in a Black-Scholes Quanto Market When Stock Price Is a Semimartingale
}

\author{
E. R. Offen, E. M. Lungu \\ University of Botswana, Gaborone, Botswana \\ Email: offen@mopipi.ub.bw, lunguem@mopipi.ub.bw
}

Received 18 April 2015; accepted 26 July 2015; published 30 July 2015

Copyright (C) 2015 by authors and Scientific Research Publishing Inc.

This work is licensed under the Creative Commons Attribution International License (CC BY). http://creativecommons.org/licenses/by/4.0/

(c) (i) Open Access

\section{Abstract}

We look at the price of the European call option in a quanto market defined on a filtered probability space $(\Omega, \mathcal{F}, \mathbb{F}, \mathbb{P})$ when the exchange rate is being modeled by the process $E_{t}=E_{0} \exp \left\{H_{t}\right\}$ where $H_{t}$ is a semimartingale. Precisely we look at an investor in a Sterling market who intends to buy a European call option in a Dollar market. The market consists of a Dollar bond, Sterling bond and and Sterling risky asset. We first of all convert the Sterling assets by using the exchange rate $E_{t}$ and later on derive an integro-differential equation that can be used to calculate the price on the option.

Keywords

Semimartingale, Hedging, Arbitrage, Contingent Claim

\section{Introduction}

This paper considers the European call option in the Black-Scholes market when the exchange-rate is a semimartingale. Specifically, we consider a problem of a Dollar investor seeking to invest in a Sterling market. Theory of exchange rates has been widely discussed (see [1]-[4]). Exchange rates change with time due to a number of factors, such as changes in fiscal and monetary policies, interest rate differentials between two countries usually resulting in revaluation or devaluation of currency. The main challenge is to construct a model which captures the dynamics of exchange rate and its effect when investments are made in different currencies. A number of models have been developed which are being modified to accommodate reality. Generally, exchange rate models fall into two major categories: Those that treat the dynamics of exchange rate as a continuous process 
and those treat exchange rates as processes with jumps. The Black-Scholes model is the most celebrated nonjump model whose dynamics are modelled by the stochastic differential equation $\mathrm{d} E_{t}=\lambda \mathrm{d} t+\sigma \mathrm{d} W_{t}$ where $E_{t}$ is the exchange rate, $\lambda$ is the drift parameter, $\sigma$ is the volatility parameter and $W_{t}$ is a Wiener process. This model assumes the logarithmic exchange which follows Brownian motion with drift. Using this as a benchmark model, other models were developed, for example, a model given by the equation $E_{t}=E_{0} \exp \left\{\lambda t+\rho \sigma W_{t}^{1}+\sqrt{1-\rho^{2}} W_{t}^{2}\right\}$ where $\left\{W_{t}^{1}, \rho W_{t}^{1}+\sqrt{1-\rho^{2}} W_{t}^{2}\right\}_{t \geq 0}$ is a pair of correlated Brownian motions with correlation co-efficient $\rho$ and $W_{t}^{1}$ is Brownian Motion driving the given asset prices [2]. It is known that jump-diffusion models are more realistic for studying the dynamics of exchange rates [3]. Dating back from the introduction of jump-diffusion process by [4] [5] as a tool for modelling the prices of options based on more general processes of underlying asset returns, jump-diffusion processes have also been widely used in modelling the dynamics of exchange rates. Empirical evidence based on simple jump-diffusion models suggests that jumps really form significant components of foreign exchange rate processes [1] [3]. As such, it is reasonable that both empirical and theoretical studies of exchange rates under uncertainty should allow for the presence of discontinuities explicitly. There has been a wide use of jump-diffusion processes to model financial time series to reflect discontinuities of asset returns. Some of the most well known jump-diffusion models for the dynamics of foreign exchange include: 1) Merton's Jump Model, 2) Conditional Heteroscedasticity and Jump model and Mean-Reversion, Conditional Heteroscedasticity and Jump model. The Merton's Jump Model is given by the stochastic differential equation $\mathrm{d} E_{t}=\left(\lambda-\alpha_{0}\right) \mathrm{d} t+\sigma \mathrm{d} W_{t}+\ln X_{t} \mathrm{~d} p_{t}(\alpha)$ where $E_{t}$ is the exchange rate, $\lambda_{t}$ is the instantaneous expected return, $\sigma$ is the instantaneous volatility of the asset's return subject to the Poisson jump not occurring, $W_{t}$ is the GaussWiener process, $p_{t}(\alpha)$ is a Poisson process which is independent and identically distributed over time, $\alpha$ is the intensity parameter of Poisson distribution, $X_{t}$ is the random jump size with $X_{t} \geq 0$ and $\mathrm{d} p_{t}(\alpha), \mathrm{d} W_{t}$ are statistically independent. This model explicitly allows for the presence of asymmetric lognormal jumps to the exchange rate. 3 ) The Conditional Heteroscedasticity and Jump model, is described by the stochastic differential equation $\mathrm{d} E_{t}=\left(\lambda-\alpha_{0}\right) \mathrm{d} t+\left(\sigma+\sigma_{1}\right) E_{t} \mathrm{~d} W_{t}+\ln X_{t} \mathrm{~d} p_{t}(\alpha)$, which is an extension of Merton's model and allows for conditional heteroscedasticity in addition to jumps. 4) Lastly, the Mean-Reversion, Conditional Heteroscedasticity and Jump model, described by the differential equation

$\mathrm{d} E_{t}=\left(\lambda-\beta E_{t}-\alpha_{0} \lambda\right) \mathrm{d} t+\left(\sigma+\sigma_{1}\right) E_{t} \mathrm{~d} W_{t}+\ln X_{t} \mathrm{~d} p_{t}(\alpha)$ where $\ln X_{t} \sim i i d N\left(\alpha_{0}, v\right)$. This model has a linear drift term in $E_{t}$ which makes it possible to capture the mean-reversion feature of the underlying process in addition to conditional heteroscedasticity and asymmetric jumps. The aim of our work is to provide further evidence for appropriateness of jump models. We do that by formulating an exchange rate model in terms of the general semimartingale on filtered probability space $(\Omega, \mathcal{F}, \mathbb{F}, \mathbb{P})$ to model the dynamics of the exchange rate $E_{t}=E_{0} \exp \left(X_{t}\right)$ where $X_{t} \in \operatorname{Sem}(\mathbb{F}, \mathbb{P})$. Our approach leads to general formulae which capture both the continuous and jump-diffusion cases models depending on whether $\Delta X_{t}=0$, the continuous model or $\Delta X_{t} \neq 0$, the jump diffusion one. The approach utilizes the specific market model called the Black-Scholes Quanto model, in other words, we consider products denominated in a currency other than those in which they are traded. Our prime interest is to price the claim $C_{T}$, which in our case is the European call option, in this market when the dynamics of the exchange rate is being modelled by the general semimartingale as described above.

\section{The Model}

We consider the quanto market model consisting of Dollar bond $B_{t}=\mathrm{e}^{r t}$, Sterling bond $D_{t}=\mathrm{e}^{u t}$, Sterling risky asset price $S_{t}=S_{0} \exp \left\{\alpha t+\sigma W_{t}\right\}$ and the Exchange rate $E_{t}=E_{0} \exp \left\{H_{t}\right\}$ on the filtered probability space $(\Omega, \mathcal{F}, \mathbb{F}, \mathbb{P})$ where $\mathbb{F}=\mathbb{F}^{S} \vee \mathbb{F}^{E}$, and $\mathbb{F}^{S}=\left\{\mathcal{F}_{t}^{S}, t \geq 0\right\}, \mathcal{F}_{t}^{S}=\sigma\left(S_{u}: 0 \leq u \leq t\right)$ is the natural filtration generated by the stock price process while $\mathbb{F}^{E}=\left\{\mathcal{F}_{t}^{E}, t \geq 0\right\}, \mathcal{F}_{t}^{E}=\sigma\left(E_{u}: 0 \leq u \leq t\right)$ is the natural filtration generated by the exchange rate process. $\mathbb{F}$ describes information about prices and the exchange rate revealed to investors. We assume that the probability space $(\Omega, \mathcal{F}, \mathbb{F}, \mathbb{P})$ satisfies the usual conditions i.e the $\sigma$-field $\mathcal{F}$ is $\mathbb{P}$-complete and every $\mathcal{F}_{t}$ contains all $\mathbb{P}$-null sets of $\mathcal{F} . W_{t}$ is the Brownian motion independent of $H_{t}$ and $H_{t} \in \operatorname{Sem}(\mathbb{F}, \mathbb{P})$ i.e $H_{t}$ is a cadlág process that admits the decomposition $H_{t}=H_{0}+A_{t}+M_{t}$ where $A_{t} \in \mathcal{V}$ (a process of bounded variation), $A_{0}=0$ and $M=M_{t} \in \mathcal{M}_{\text {lock}}$. For a dollar investor in the quanto market defined in this problem, we want to find what is the price of the European 
call option having strike price $K$ and strike time $T$ ?

\section{Converting into Dollars}

Since our asset is in Sterling, we need first of all to find the Dollar equivalent of this asset. For convenience sake we let $S_{0}=E_{0}=1$. $Y_{t}$ be the dollar value of the Sterling asset price given by

$$
Y_{t}=E_{t} \cdot S_{t}
$$

Define

$$
Y_{t}=\exp \left\{X_{t}\right\}
$$

where

$$
X_{t}=\alpha t+\sigma W_{t}+H_{t}
$$

$X_{t}$ is a semimartingale since it is the sum of two semimartingales $\alpha t+\sigma W_{t}$ and $H_{t}$. This in turn implies that $Y_{t}$ is a semimartingale. Let

$$
Y_{t}=f\left(X_{t}\right)=\exp \left(X_{t}\right)
$$

Then, using Ito's formula for semimartingales [6] [7], the dynamics of the dollar value for the Sterling risky asset is

$$
\mathrm{d} Y_{t}=Y_{t-} \mathrm{d} \hat{X}_{t}
$$

where

$$
\hat{X}_{t}=X_{t}+\frac{1}{2}\left\langle X^{c}\right\rangle_{t}+\sum_{0<s \leq t}\left(\mathrm{e}^{\Delta X_{s}}-1-\Delta X_{s}\right) .
$$

(see Appendix). Let $X_{t}=\alpha t+W_{t}+H_{t}$ so that $X_{t}=V_{t}+H_{t}$ then $\Delta X_{t}=\Delta V_{t}+\Delta H_{t}$ and

$$
X_{t}^{c}=V_{t}^{c}+H_{t}^{c} \text {. }
$$

$V_{t}$ being a semimartingale has the form

$$
V_{t}=V_{0}+A_{t}+M_{t}^{v}
$$

It is important to take note that superscript $v$ in $M_{t}^{v}$ is used to stress that $M_{t}^{v}$ is the martingale part for process $V_{t}$ which in this case

$$
M_{t}^{v}=\sigma W_{t} .
$$

Since $M_{t}^{v}$ is a local martingale, it can be decomposed as

$$
M_{t}^{v}=M_{t}^{v c}+M_{t}^{v d}
$$

where $M_{t}^{v c}$ is the continuous part and $M_{t}^{v d}$ is the discontinuous part of $M_{t}^{v}$. For more details and a proof of Equation (8) see [8]. Since $\Delta \sigma W_{t}=0$, we have $M_{t}^{v d}=0$. From Equation (6), we have $V_{t}^{c}=\sigma W$. Similarly if we let $H_{t}^{c}$ to be the continuous martingale part of $H_{t}$ then

$$
X_{t}^{c}=\sigma W_{t}+H_{t}^{c}
$$

Now, using the bilinear property of sharp bracket process [9], we obtain

$$
\begin{aligned}
\left\langle X^{c}\right\rangle_{t} & =\left\langle\sigma W+H^{c}\right\rangle_{t} \\
& =\sigma^{2}\langle W, W\rangle_{t}+2\left\langle\sigma W, H^{c}\right\rangle_{t}+\left\langle H^{c}, H^{c}\right\rangle_{t} \\
& =\sigma^{2} t+\left\langle H^{c}, H^{c}\right\rangle_{t} \\
& =\sigma^{2} t+\left\langle H^{c}\right\rangle_{t} .
\end{aligned}
$$


We further note that

$$
\begin{aligned}
\Delta V_{t} & =V_{t}-V_{t_{-}} \\
& =\left[\mu t+\sigma W_{t}\right]-\left[\mu\left(t_{-}\right)+\sigma W_{t_{-}}\right] \\
& =\left(\mu t-\mu\left(t_{-}\right)\right)+\left(\sigma W_{t}-\sigma W_{t_{-}}\right) \\
& =0
\end{aligned}
$$

This is true because $\mu t$, and $\sigma W_{t}$ are continuous processes. It then follows that

$$
\Delta X_{t}=\Delta H_{t} .
$$

Substituting $X_{t}$ and Equations (3), (10), (11) into Equation (5) we have

$$
\hat{X}_{t}=\alpha t+\sigma W_{t}+H_{t}+\frac{1}{2}\left(\sigma^{2} t+\left\langle H^{c}\right\rangle_{t}\right)+\sum_{0<s \leq t}\left(\mathrm{e}^{\Delta H_{s}}-1-\Delta H_{s}\right) .
$$

Similarly, let $Z_{t}$ be the dollar value of the Sterling bond given by

$$
Z_{t}=E_{t} \cdot D_{t}=\exp \left\{u t+H_{t}\right\} .
$$

Let

$$
Z_{t}=g\left(u t+H_{t}\right)=\mathrm{e}^{u t+H_{t}} .
$$

It clearly follows that

$$
\begin{aligned}
Z_{t}= & \mathrm{e}^{X_{0}}+\int_{0}^{t}\left(\mathrm{e}^{u s_{-}+H_{s_{-}}}\right)^{\prime} \mathrm{d}\left(u s+H_{s}\right)+\frac{1}{2} \int_{0}^{t}\left(\mathrm{e}^{u s_{-}+H_{s_{-}}}\right)^{\prime \prime \prime} \mathrm{d}\left\langle u s+H_{s}^{c}\right\rangle \\
& +\sum_{0<s \leq t}\left[\mathrm{e}^{u s+H_{s}}-\mathrm{e}^{u s_{-}+H_{s_{-}}}-\left(\mathrm{e}^{u s_{-}+H_{s_{-}}}\right)^{\prime} \Delta\left(u s+H_{s}\right)\right] .
\end{aligned}
$$

Noting that

$$
\begin{aligned}
\Delta\left(u s+H_{s}\right) & =\left(u s+H_{s}\right)-\left(u s_{-}+H_{s_{-}}\right) \\
& =\left(u s-u s_{-}\right)+\left(H_{s}-H_{s_{-}}\right) \\
& =H_{s}-H_{s_{-}} \\
& =\Delta H_{s} .
\end{aligned}
$$

Note that Equation (15) follows because $u s$ is a continuous function so that $u s=u s_{-}$. The continuous martingale part of $u s+H_{s}$ is

$$
\left(u s+H_{s}\right)^{c}=H_{s}^{c},
$$

Hence

$$
\left\langle\left(u s+H_{s}\right)^{c}\right\rangle=\left\langle H^{c}\right\rangle_{s} .
$$

$Z_{t}$ can now be written as

$$
\begin{aligned}
Z_{t}= & \mathrm{e}^{H_{0}}+\int_{0}^{t} \mathrm{e}^{u s+H_{s-}} \mathrm{d}\left(u s+H_{s}\right)+\frac{1}{2} \int_{0}^{t} \mathrm{e}^{u s+H_{s-}} \mathrm{d}\left\langle H^{c}\right\rangle_{s} \\
& +\sum_{0 \leq s \leq t}\left[\mathrm{e}^{u s+X_{s}}-\mathrm{e}^{u s+H_{s-}}-\mathrm{e}^{u s+H_{s-}} \Delta H_{s}\right] .
\end{aligned}
$$

Its differential form, the dynamics of the dollar value of the Sterling bond can be written as 


$$
\begin{aligned}
\mathrm{d} Z_{t} & =\mathrm{e}^{u t+H_{t-}} \mathrm{d}\left(u t+H_{t}\right)+\frac{1}{2} \mathrm{e}^{u t+H_{t-}} \mathrm{d}\left\langle H^{c}\right\rangle_{t}+\mathrm{e}^{u t+H_{t}}-\mathrm{e}^{u t+H_{t-}}-\mathrm{e}^{u t+H_{t-}} \Delta H_{t} \\
& =\mathrm{e}^{u t+H_{t-}} \mathrm{d}\left(u t+H_{t}\right)+\frac{1}{2} \mathrm{e}^{u t+H_{t-}} \mathrm{d}\left\langle H^{c}\right\rangle_{t}+\mathrm{e}^{u t+H_{t-}+\Delta\left(u t+H_{t}\right)}+\mathrm{e}^{u t+H_{t-}}-\mathrm{e}^{u t+H_{t-}} \Delta H_{t} \\
& =\mathrm{e}^{u t+H_{t-}} \mathrm{d}\left[u t+H_{t}+\frac{1}{2}\left\langle H^{c}\right\rangle_{t}+\sum_{0<s \leq t}\left[\mathrm{e}^{\Delta\left(u s+H_{s}\right)}-1-\Delta H_{s}\right]\right]
\end{aligned}
$$

Which can be written as

$$
\mathrm{d} Z_{t}=Z_{t_{-}} \mathrm{d} \hat{K}_{t}
$$

where

$$
\hat{K}_{t}=u t+H_{t}+\frac{1}{2}\left\langle H^{c}\right\rangle_{t}+\sum_{0<s \leq t}\left(\mathrm{e}^{\Delta H_{s}}-1-\Delta H_{s}\right)
$$

And

$$
Z_{t_{-}}=\mathrm{e}^{u t+H_{t_{-}}} .
$$

For our analysis, we need to express the decompositions $\hat{X}_{t}$ (Equation (12)) and $\hat{K}_{t}$ (Equation (17)), in stochastic integral form, hence we use the random measure of jumps.

In our case, we have

$$
X_{t}=V_{t}+H_{t}
$$

And from Equation (10) we have

$$
\left\langle X^{c}\right\rangle_{t}=\sigma_{1}^{2} t+\left\langle H^{c}\right\rangle_{t}
$$

Hence, process of bounded variation $B(\phi)$ can be express as

$$
B(\phi)_{t}=A(\phi)_{t}+D(\phi)_{t}=\alpha t+D(\phi)_{t}, H_{0}=V_{0}+X_{0}=X_{0}
$$

where $A$ is as defined in Equation (6) and $D$ is the process of bounded variation for the semimartingale $H_{t}$. We can express these results in canonical form by using the random measure of jumps (see [7]). From Equation (5),

$$
\begin{aligned}
\hat{X}_{t} & =X_{t}+\frac{1}{2}\left\langle X^{c}\right\rangle_{t}+\sum_{0<s \leq t}\left(\mathrm{e}^{\Delta X_{s}}-1-\Delta X_{s}\right) \\
& =X_{t}+\frac{1}{2}\left\langle X^{c}\right\rangle_{t}+\left(\mathrm{e}^{x}-1-x\right) \star \mu \\
& =X_{0}+B(\phi)_{t}+X_{t}^{c}+\frac{1}{2}\left\langle X^{c}\right\rangle_{t}+\phi(x) \star(\mu-v)+x-\phi(x) \star \mu+\left(\mathrm{e}^{x}-1-x\right) \star \mu \\
& =X_{0}+B(\phi)_{t}+X_{t}^{c}+\frac{1}{2}\left\langle X^{c}\right\rangle_{t}+\phi(x) \star(\mu-v)+\left(\mathrm{e}^{x}-1-\phi(x)\right) \star \mu .
\end{aligned}
$$

Now, if we assume that $\left(\left|\mathrm{e}^{x}-1-\phi(x)\right| * \mu \in \mathcal{A}_{\text {loc }}\right.$, then

$$
\begin{aligned}
\hat{X}_{t}= & X_{0}+B(\phi)_{t}+X_{t}^{c}+\frac{1}{2}\left\langle X^{c}\right\rangle_{t}+\left(\mathrm{e}^{x}-1-\phi(x)\right) \star v \\
& +\left(\mathrm{e}^{x}-1-\phi(x)\right) \star(\mu-v)+\phi(x) \star(\mu-v) \\
= & X_{0}+\tilde{B}_{t}+X_{t}^{c}+\left(\mathrm{e}^{x}-1\right) \star(\mu-v)
\end{aligned}
$$

where

$$
\tilde{B}_{t}=B(\phi)_{t}+\frac{1}{2}\left\langle X^{c}\right\rangle_{t}+\left(\mathrm{e}^{x}-1-\phi(x)\right) \star v .
$$

Now if we substitute Equations (9) (10) and (19) into Equation (21) we obtain 


$$
\hat{X}_{t}=H_{0}+G_{t}+\sigma W_{t}+H_{t}^{c}+\left(\mathrm{e}^{x}-1\right) \star(\mu-v)
$$

where

$$
G_{t}=\alpha t+D(\phi)_{t}+\frac{1}{2}\left(\sigma^{2} t+\left\langle H^{c}\right\rangle_{t}\right)+\left(\mathrm{e}^{x}-1-\phi(x)\right) \star v,
$$

i.e.

$$
\hat{X}_{t}=\hat{X}_{t}^{*}+G_{t}
$$

where $\hat{X}_{t}^{*} \in \mathcal{M}_{\text {loc }}$ and $G_{t}$ is a predictable process. Similarly from Equation (13)

If we set $K_{t}=u t+H_{t}$ with $\phi_{*}$ as a truncation function, we have $K_{t}^{c}=H_{t}^{c}$,

$\left\langle K^{c}\right\rangle_{t}=\left\langle H^{c}\right\rangle_{t}, \quad K_{0}=H_{0}$ and together with Equation (17),

$$
\hat{K}_{t}=H_{0}+R_{t}+H_{t}^{c}+\left(\mathrm{e}^{x}-1\right) \star(\mu-v)
$$

where

$$
R_{t}=u t+D\left(\phi_{*}\right)_{t}+\frac{1}{2}\left\langle H^{c}\right\rangle_{t}+\left(\mathrm{e}^{x}-1-\phi_{*}(x)\right) \star v .
$$

This means the dynamics in our market model are modeled by the equations can also be presented by the eququations

$$
\begin{aligned}
& \mathrm{d} \mathbf{B}_{t}=r d t \\
& \mathrm{~d} Z_{t}=Z_{t-} d \hat{K}_{t} \\
& \mathrm{~d} Y_{t}=Y_{t-} d \hat{X}_{t}
\end{aligned}
$$

where $\hat{X}_{t}$ and $\hat{K}_{t}$ are as defined in Equations (21) and (26) respectively. Before we proceed we need to show that our market model defined by the system of Equation (27) does not entertain arbitrage opportunities.

\section{Arbitrage}

A question we must ask before we proceed is whether the market (27) allows arbitrage opportunities or not. In this market, an investment strategy or portfolio is a predictable process

$$
\psi=(\vartheta, \varphi, \theta) \in \mathbb{R}^{3}
$$

Such that

$$
\int_{0}^{T}\left\{\vartheta_{s}^{2}+\varphi_{s}^{2}+\theta_{s}^{2}\right\} \mathrm{d} s \leq \infty \text { a.s } \mathbb{P}
$$

$\vartheta_{t}, \varphi_{t}, \theta_{t}$ denote the fractions of total wealth invested in $\mathbf{B}, Z$, and $Y$ respectively at time $t$. The condition in inequality (29) ensures that the integrals $\int_{0}^{t} \varphi_{s} \mathrm{~d} \tilde{Z}_{s}+\int_{0}^{t} \theta_{s} \mathrm{~d} \tilde{Y}_{s}$ make sence and are is a martingales.

Let

$$
V_{t}^{\psi}=\vartheta_{t} \mathbf{B}_{t}+\varphi Z_{t}+\theta_{t} Y_{t} \quad 0 \leq t \leq T
$$

be the worth the worth process. We need to know if our portfolio $\psi$ is self-financing. A portfolios is self-financing if $V_{t}^{\psi}$ can also be written as

$$
V^{\psi}(t)=V_{0}^{\psi}+\int_{0}^{t} \vartheta_{s} \mathrm{~d} \mathbf{B}_{s}+\int_{0}^{t} \varphi_{s} \mathrm{~d} Z_{s}+\int_{0}^{t} \theta_{s} \mathrm{~d} Y_{s}
$$

or in differential form, if

$$
\mathrm{d} V^{\psi}(t)=\vartheta_{t} \mathrm{~d} \mathbf{B}_{t}+\varphi_{t} \mathrm{~d} Z_{t}+\theta_{t} \mathrm{~d} Y_{t} .
$$

Equations (31) and (32) imply that the portfolio is self-financing if changes in the value of the portfolio on an infinitesimal interval are due entirely to the changes in value of assets and not to an injection (or removal) of wealth from outside. 
To show that our portfolio is self-financing, we use Lemma (5.1.3) in [2]. According to [2], $\psi$ is a selffinancing portfolio if

$$
\tilde{V}^{\psi}(t)=\tilde{V}_{0}^{\psi}+\int_{0}^{t} \varphi_{s} \mathrm{~d} \tilde{Z}_{s}+\int_{0}^{t} \theta_{s} \mathrm{~d} \tilde{Y}_{s}
$$

where $\tilde{V}^{\psi}(t)=\mathrm{e}^{-r t} V^{\psi}(t)$, the discounted value of the wealth process.

$$
\begin{aligned}
\mathrm{d} \tilde{V}^{\psi}(t) & =\mathrm{de}^{-r t} V^{\psi}(t) \\
& =-r \mathrm{e}^{-r t} V^{\psi}(t)+\mathrm{e}^{-r t} \mathrm{~d} V^{\psi}(t) \\
& =-r \mathrm{e}^{-r t}\left(\vartheta_{t} \mathbf{B}_{t}+\varphi Z_{t}+\theta_{t} Y_{t}\right)+\mathrm{e}^{-r t}\left(r \vartheta_{t} \mathrm{~d} \mathbf{B}_{t}+\varphi_{t} \mathrm{~d} Z_{t}+\theta_{t} \mathrm{~d} Y_{t}\right) \\
& =-r \mathrm{e}^{-r t}\left(\vartheta_{t} \mathrm{e}^{r t}+\varphi Z_{t}+\theta_{t} Y_{t}\right)+\mathrm{e}^{-r t}\left(\vartheta_{t} \mathrm{e}^{r t}+\varphi_{t} \mathrm{~d} Z_{t}+\theta_{t} \mathrm{~d} Y_{t}\right) \\
& =-r \mathrm{e}^{-r t} \varphi Z_{t}-r \mathrm{e}^{-r t} \theta_{t} Y_{t}+\mathrm{e}^{-r t} \varphi_{t} \mathrm{~d} Z_{t}+\mathrm{e}^{-r t} \theta_{t} \mathrm{~d} Y_{t} \\
& =-r \mathrm{e}^{-r t} \theta_{t} Z_{t}+\mathrm{e}^{-r t} \theta_{t} \mathrm{~d} Z_{t}-r \mathrm{e}^{-r t} \varphi Y_{t}+\mathrm{e}^{-r t} \varphi_{t} \mathrm{~d} Y_{t} \\
& =\theta_{t} \mathrm{~d}\left(\mathrm{e}^{-r t} Z_{t}\right)+\varphi_{t} \mathrm{~d}\left(\mathrm{e}^{-r t} Y_{t}\right)
\end{aligned}
$$

This means

$$
\begin{aligned}
& \mathrm{d} \tilde{V}_{t}^{\psi} t=\theta_{t} \mathrm{~d}\left(\mathrm{e}^{-r t} Z_{t}\right)+\varphi_{t} \mathrm{~d}\left(\mathrm{e}^{-r t} Y_{t}\right) \\
& \tilde{V}_{t}^{\psi}=\tilde{V}_{0}^{\psi}+\int_{0}^{t} \theta_{s} \mathrm{~d} \tilde{Z}_{s}+\int_{0}^{t} \varphi_{s} \mathrm{~d} \tilde{Y}_{s},
\end{aligned}
$$

Satisfying Equation (33). Hence $\psi$ is a self-financing portfolio. If in additional to this to (34), $\left\{V_{t}^{\psi}\right\}_{t \in[0, T]}$ as defined in Equation (34) above is lower bounded, then $\psi_{t}$ is said to be admissible. It is written as $\psi \in \mathcal{A}_{0}$.

If $\psi_{t}$ is not admissible, it is not hard to construct doubling strategies, that is, a portfolio that attains arbitrarily large values of wealth with probability one when $t=T$, starting with zero initial capital at $t=0$, a situation we are avoiding. We first of all show that in this market, $\psi_{t}$ is an admissible strategy. The dynamic $\mathrm{d} Z_{t}$ for the dollar value of the sterling bond is given by the equation

$$
\begin{aligned}
\mathrm{d} Z_{t} & =Z_{t-} \mathrm{d} \hat{K}_{t-} \\
& =Z_{t-}\left[u \mathrm{~d} t+d D(\phi)_{t}+\mathrm{d} H_{t}^{c}+\frac{1}{2} \mathrm{~d}\left\langle H^{c}\right\rangle_{t}+\mathrm{d}\left(\left(\mathrm{e}^{x}-1\right) \star(\mu-v)\right)+\mathrm{d}\left(\mathrm{e}^{x}-1-\phi(x)\right) \star \mu\right] \\
& =Z_{t-}\left[u \mathrm{~d} t+\mathrm{d} D(\phi)_{t}+\mathrm{d} H_{t}^{c}+\frac{1}{2} \mathrm{~d}\left\langle H^{c}\right\rangle_{t}+\int_{-\infty}^{\infty}\left(\mathrm{e}^{x}-1\right) \mathrm{d}(\mu-v)+\int_{-\infty}^{\infty}\left(\mathrm{e}^{x}-1-\phi(x)\right) \mathrm{d} \mu\right]
\end{aligned}
$$

Similarly, the dynamic $d Y_{t}$ for the dollar value of the Sterling risky asset is given by the equation

$$
\begin{aligned}
\mathrm{d} Y_{t}= & Y_{t-} \mathrm{d} \hat{X}_{t} \\
= & Y_{t-}\left[\alpha \mathrm{d} t+\mathrm{d} D(\phi)_{t}+\sigma \mathrm{d} W_{t}+\mathrm{d} H_{t}^{c}+\frac{1}{2} \sigma^{2} \mathrm{~d} t\right. \\
& \left.+\frac{1}{2} \mathrm{~d}\left\langle H^{c}\right\rangle_{t}+\mathrm{d}\left(\left(\mathrm{e}^{x}-1\right) \square(\mu-v)\right)+\mathrm{d}\left(\mathrm{e}^{x}-1-\phi(x) \square \mu\right)\right] \\
= & Y_{t-}\left[\alpha \mathrm{d} t+\mathrm{d} D(\phi)_{t}+\sigma \mathrm{d} W_{t}+\mathrm{d} H_{t}^{c}+\frac{1}{2} \sigma^{2} \mathrm{~d} t\right. \\
& \left.+\frac{1}{2} \mathrm{~d}\left\langle H^{c}\right\rangle_{t}+\int_{-\infty}^{\infty}\left(\mathrm{e}^{x}-1\right) \mathrm{d}(\mu-v)+\int_{-\infty}^{\infty}\left(\mathrm{e}^{x}-1-\phi(x)\right) \mathrm{d} \mu\right]
\end{aligned}
$$

From Equation (30)

$$
\vartheta_{t}=\mathrm{e}^{-r t}\left(V_{t}^{\psi}-\varphi Z_{t}-\theta_{t} Y_{t}\right)
$$

Since the portfolio $\psi$ is self-financing (Equation (32)), then 


$$
\begin{aligned}
\mathrm{d} V^{\psi}(t)= & \vartheta_{t} \mathrm{~d} \mathbf{B}_{t}+\varphi_{t} \mathrm{~d} Z_{t}+\theta_{t} \mathrm{~d} Y_{t} \\
= & r \vartheta_{t} \mathbf{B}_{t} \mathrm{~d} t+\varphi_{t} \mathrm{~d} Z_{t}+\theta_{t} \mathrm{~d} Y_{t} \\
= & r e^{r t} \cdot \mathrm{e}^{-r t} \mathrm{~d} t\left(V^{\psi}(t)-\varphi_{t} Z_{t}-\theta_{t} Y_{t}\right)+\varphi \mathrm{d} Z_{t}+\theta_{t} \mathrm{~d} Y_{t} \\
= & r V_{t}^{\psi} \mathrm{d} t-r \varphi_{t} Z_{t} \mathrm{~d} t-r \theta_{t} Y_{t} \mathrm{~d} t \\
& +\varphi_{t} Z_{t-}\left[u \mathrm{~d} t+\mathrm{d} D(\phi)_{t}+\mathrm{d} X_{t}^{c}+\frac{1}{2} \mathrm{~d}\left\langle H^{c}\right\rangle_{t}+\int_{-\infty}^{\infty}\left(\mathrm{e}^{x}-1\right) d(\mu-v)+\int_{-\infty}^{\infty}\left(\mathrm{e}^{x}-1-\phi(x)\right) \mathrm{d} \mu\right] \\
& +\theta_{t} Y_{t-}\left[\alpha \mathrm{d} t+\mathrm{d} C(\phi)_{t}+\sigma \mathrm{d} W_{t}+\mathrm{d} H_{t}^{c}+\frac{1}{2} \sigma^{2} \mathrm{~d} t+\frac{1}{2} \mathrm{~d}\left\langle X^{c}\right\rangle_{t}+\int_{-\infty}^{\infty}\left(\mathrm{e}^{x}-1\right) \mathrm{d}(\mu-v)+\int_{-\infty}^{\infty}\left(\mathrm{e}^{x}-1-\phi(x)\right) \mathrm{d} \mu\right]
\end{aligned}
$$

Then

$$
\begin{aligned}
& \mathrm{e}^{-r t} \mathrm{~d}\left(V_{t}^{\psi}\right)-r \mathrm{e}^{-r t} V_{t}^{\psi} \mathrm{d} t \\
& =\mathrm{e}^{-r t} \varphi_{t} Z_{t-}\left[(u-r) \mathrm{d} t+\mathrm{d} D(\phi)_{t}+\mathrm{d} H_{t}^{c}+\frac{1}{2} \mathrm{~d}\left\langle H^{c}\right\rangle_{t}+\int_{-\infty}^{\infty}\left(\mathrm{e}^{x}-1\right) \mathrm{d}(\mu-v)+\int_{-\infty}^{\infty}\left(\mathrm{e}^{x}-1-\phi(x)\right) \mathrm{d} \mu\right] \\
& +\mathrm{e}^{-r t} \theta_{t} Y_{t-}\left[(\alpha-r) \mathrm{d} t+\mathrm{d} C(\phi)_{t}+\sigma \mathrm{d} W_{t}+\mathrm{d} H_{t}^{c}+\frac{1}{2} \sigma^{2} \mathrm{~d} t\right. \\
& \left.+\frac{1}{2} \mathrm{~d}\left\langle H^{c}\right\rangle_{t}+\int_{-\infty}^{\infty}\left(\mathrm{e}^{x}-1\right) \mathrm{d}(\mu-v)+\int_{-\infty}^{\infty}\left(\mathrm{e}^{x}-1-\phi(x)\right) \mathrm{d} \mu\right]
\end{aligned}
$$

This means the differential form of of the dynamics of the discounted wealth process is

$$
\begin{aligned}
& \mathrm{d}\left(\mathrm{e}^{-r t} V_{t}^{\psi}\right) \\
& =\varphi_{t} Z_{t-}\left[(u-r) \mathrm{d} t+\mathrm{d} D(\phi)_{t}+\mathrm{d} H_{t}^{c}+\frac{1}{2} \mathrm{~d}\left\langle H^{c}\right\rangle_{t}+\int_{-\infty}^{\infty}\left(\mathrm{e}^{x}-1\right) \mathrm{d}(\mu-v)+\int_{-\infty}^{\infty}\left(\mathrm{e}^{x}-1-\phi(x)\right) \mathrm{d} \mu\right] \\
& +\theta_{t} Y_{t-}\left[(\alpha-r) \mathrm{d} t+\mathrm{d} C(\phi)_{t}+\sigma \mathrm{d} W_{t}+\mathrm{d} H_{t}^{c}+\frac{1}{2} \sigma^{2} \mathrm{~d} t+\frac{1}{2} \mathrm{~d}\left\langle H^{c}\right\rangle_{t}+\int_{-\infty}^{\infty}\left(\mathrm{e}^{x}-1\right) \mathrm{d}(\mu-v)+\int_{-\infty}^{\infty}\left(\mathrm{e}^{x}-1-\phi(x)\right) \mathrm{d} \mu\right]
\end{aligned}
$$

Hence the discounted wealth process will be

$$
\begin{aligned}
\mathrm{e}^{-r t} V_{t}^{\psi} & \\
= & V_{0}^{\psi}+\int_{0}^{t} \mathrm{e}^{-r s} \varphi_{s} Z_{s-}\left\{(u-r) \mathrm{d} s+\mathrm{d} D(\phi)_{s}+\mathrm{d} H_{s}^{c}+\frac{1}{2} \mathrm{~d}\left\langle X^{c}\right\rangle_{s}+\int_{-\infty}^{\infty}\left(\mathrm{e}^{x}-1\right) \mathrm{d}(\mu-v)+\int_{-\infty}^{\infty}\left(\mathrm{e}^{x}-1-\phi(x)\right) \mathrm{d} \mu\right\} \\
& +\int_{0}^{t} \mathrm{e}^{-r s} \theta_{s} Y_{s-}\left\{(\alpha-r) \mathrm{d} s+\mathrm{d} C(\phi)_{t}+\sigma \mathrm{d} W_{s}+\mathrm{d} H_{s}^{c}+\frac{1}{2} \sigma^{2} \mathrm{~d} s\right. \\
& \left.+\frac{1}{2} \mathrm{~d}\left\langle H^{c}\right\rangle_{s}+\int_{-\infty}^{\infty}\left(\mathrm{e}^{x}-1\right) \mathrm{d}(\mu-v)+\int_{-\infty}^{\infty}\left(\mathrm{e}^{x}-1-\phi(x)\right) \mathrm{d} \mu\right\}
\end{aligned}
$$

From the above equations, $\mathrm{e}^{-r t} V^{\psi}(t)$ is a lower bounded process. It follows that $V^{\psi}(t)$ is lower bounded implying that $\psi$ is an admissible strategy.

Definition 1 A portfolio $\psi_{t} \in \mathcal{A}_{0}$ is called arbitrage if

$$
V_{0}^{\psi}=0, V_{T}^{\psi} \geq 0 \text { and } \mathbb{P}\left[V_{T}^{\psi}>0\right]>0 .
$$

Since the portfolio $\psi$ in our market is admissible, we really claim there are no arbitrage opportunities in the market. The following discussion is a build up towards the proof to justify this claim.

\subsection{Converting $Y_{t}$ into a Martingale}

Our stock price process as described in Equation (27) is a semimartingale. To use the martingale approach, we need to convert the price process into a martingale by finding another probability space $\mathbb{P}^{*}$ equivalent to our 
probability $\mathbb{P}$ so that $Y_{t}$ becomes a martingale. Now we consider our price process

$$
\mathrm{d} Y_{t}=Y_{-} \mathrm{d} \hat{X}_{t}
$$

(see Equation (27)). $Y_{t}=\mathcal{E}(\tilde{X})_{t}$ and with Equation (2), we can say

$$
\mathrm{e}^{X_{t}}=\mathcal{E}(\tilde{X})_{t} 0 \leq t \leq T
$$

This means our price process is a local martingale iff $\mathrm{e}^{X_{t}}\left(\mathcal{E}(\tilde{X})_{t}\right)$ is a local martingale. Now $\mathrm{e}^{X_{t}}\left(\mathcal{E}(\tilde{X})_{t}\right)$ becomes a local martingale through the change of probability measure theorem for semimartingales i.e. we find another measure $\mathbb{P}^{*} \sim \mathbb{P}$ under which $Y_{t}$ becomes a martingale. To achieve this, we consider the following:

Suppose we have the triplet $(B, C, v)$ for a semimartingale $X$ under measure $\mathbb{P}$ what are the triplets $\left(B^{*}, C^{*}, v^{*}\right)$ for semimartingale under a new probability measure $\mathbb{P}^{*}$ ?

Using the Gisanov's Theorem for semimartingales,

$$
\begin{aligned}
& B^{*}=B+\beta \cdot C+\phi(x)(\mathbb{Y}-1) \star v, \\
& C^{*}=C, \\
& v^{*}=\mathbb{Y} \cdot v
\end{aligned}
$$

where $\beta$ satisfies the equation

$$
\left\langle Y^{c}, X^{c}\right\rangle=\left(Y_{-} \beta\right) \cdot C
$$

And $\mathbb{Y}=\mathbb{Y}(\omega, t, x)$ is defined by the equation

$$
\mathbb{Y}=M_{\mu^{X}}^{\mathbb{P}}\left(\frac{Y}{Y_{-}} \mid \tilde{\mathcal{P}}\right)
$$

where $M_{\mu^{X}}^{\mathbb{P}}=\mu^{X}(\omega, \mathrm{d} t, \mathrm{~d} x) \mathbb{P}(\mathrm{d} \omega)$ is the positive measure on $(\Omega \times[0, T] \times \mathbb{R}, \mathcal{F} \otimes \mathcal{B}([0, T]) \otimes \mathcal{B}(\mathbb{R}))$ defined by

$$
M_{\mu^{X}}^{\mathbb{P}}(W)=\mathbb{E}\left(W \star \mu^{X}\right)_{T}
$$

where $W=W(\omega, t, x)$ is a nonnegative function on $\Omega \times[0, T] \times \mathbb{R} \quad$ (for $\tilde{\mathcal{P}}$ see [8]). $M_{\mu^{X}}^{\mathbb{P}}\left(\frac{Y}{Y_{-}} \mid \tilde{\mathcal{P}}\right)$ is, by definition, the $M_{\mu^{X}}^{\mathbb{P}}$-a.s unique $\tilde{\mathcal{P}}$-measurable function $\mathbb{Y}$ with property

$$
M_{\mu^{X}}^{\mathbb{P}}\left(\frac{Y}{Y_{-}} V\right)=M_{\mu^{X}}^{\mathbb{P}}(\mathbb{Y} V)
$$

For all non-negative $\tilde{\mathbb{P}}$-measurable functions $V=V(\omega ; t, x)$. This means that under measure $\mathbb{P}^{*}$, the semimartingale process $\hat{X}$ evolves according to the equation

$$
\hat{X}_{t}=X_{0}+G_{t}^{*}+\sigma W_{t}^{*}+H_{t}^{c}+\left(\mathrm{e}^{x}-1\right) \star(\mu-\tilde{v})
$$

where

$$
G_{t}^{*}=B^{*}(\phi)_{t}+\frac{1}{2}\left(\sigma^{2} t+\left\langle H^{c}\right\rangle_{t}\right)+\left(\mathrm{e}^{x}-1-\phi(x)\right) \star v^{*}
$$

And

$$
B^{*}(\phi)_{t}=B(\phi)_{t}+\beta\left(\sigma W_{t}^{*}+H_{t}^{c}\right)+\phi(x)(\mathbb{Y}-1) \star v^{*},
$$

$W_{t}^{*}=W_{t}-\int_{0}^{t} \sqrt{c} \beta_{s} \mathrm{~d} s$ is a $\mathbb{P}^{*}$-Brownian motion [10].

We start by finding the values $\beta$ and $\mathbb{Y}$ for our case. To do this we first find $Y_{t}^{c}$, the continuous martingale part for the risky asset process $Y_{t}$, as described by Equation (2). Using Itó's formula for semimartingales, we have 


$$
\begin{aligned}
\mathrm{e}^{H_{t}+\sigma W_{t}+\alpha t}= & +\int_{0} \mathrm{e}^{H_{s-}+\sigma W_{s}+\alpha s}\left(\mathrm{~d} H_{s}+\sigma \mathrm{d} W_{s}+\alpha \mathrm{d} s\right)+\frac{1}{2} \int_{0} \mathrm{e}^{H_{s-}+\sigma W_{s}+\alpha s}\left(\mathrm{~d}\left\langle H^{c}\right\rangle_{s}+\sigma^{2} \mathrm{~d} s\right) \\
& +\int_{0} \int_{\mathbb{R}}\left(\mathrm{e}^{H_{s-}+\sigma W_{s}+\alpha s+x}-\mathrm{e}^{H_{s-}+\sigma W_{s}+\alpha s}-\mathrm{e}^{H_{s-}+\sigma W_{s}+\alpha s} x\right) \mu^{X}(\mathrm{~d} s, \mathrm{~d} x) \\
= & 1+\int_{0} \mathrm{e}^{H_{s-}+\sigma W_{s}+\alpha t}\left(\mathrm{~d} D_{s}(\phi)+\alpha \mathrm{d} s\right)+\frac{1}{2} \int_{0} \mathrm{e}^{H_{s-}+\sigma W_{s}+\alpha s}\left(\mathrm{~d}\left\langle H^{c}\right\rangle_{s}+\sigma^{2} \mathrm{~d} s\right) \\
& \int_{0} \int_{\mathbb{R}}\left(\mathrm{e}^{H_{s-}+\sigma W_{s}+\alpha s+x}-\mathrm{e}^{H_{s-}+\sigma W_{s}+\alpha s}-\mathrm{e}^{H_{s-}+\sigma W_{s}+\alpha s} \phi(x)\right) \mu(\mathrm{d} s, \mathrm{~d} x) \\
& \int_{0} \mathrm{e}^{H_{s-}+\sigma W_{s}+\alpha s}\left(\mathrm{~d} H_{s}^{c}+\sigma \mathrm{d} W_{t}\right)+\int_{0} \int_{\mathbb{R}} \mathrm{e}^{H_{s-}+\sigma W_{s}+\alpha s} \phi(x)(\mu-v)(\mathrm{d} s, \mathrm{~d} x)
\end{aligned}
$$

It is easy to see that the continuous part of the semimartingale $e^{H_{t}+\sigma W_{t}+\alpha t}$ is given by

$$
\left(\mathrm{e}^{H_{t}+\sigma W_{t}+\alpha t}\right)^{c}=\int_{0} \mathrm{e}^{H_{s-}+\sigma W_{t}+\alpha t}\left(\mathrm{~d} H_{t}^{c}+\sigma \mathrm{d} W_{t}\right)
$$

Hint: in our calculations, we have made use of Equation (2) and the canonical decomposition of the semimartingale hence the differential form of $X_{t}$ in our case can be written as

$$
\mathrm{d} X_{t}=\mathrm{d} D(\phi)_{t}+\alpha \mathrm{d} t+\mathrm{d} H_{t}^{c}+\sigma \mathrm{d} W_{t}+\int_{\mathbb{R}} \phi(x) \mathrm{d} \star(\mu-v)+\int_{\mathbb{R}} \mathrm{d}(x-\phi(x)) \star \mu .
$$

From Equation (47) and using properties of conditional quadratic variation process for stochastic integrals with respect to semimartingales

$$
\begin{aligned}
\left\langle Y^{c}, X^{c}\right\rangle_{t} & =\left\langle\left(\mathrm{e}^{X_{t}+\sigma W_{t}+\alpha t}\right)^{c}, H^{c}+\sigma W_{t}\right\rangle=\left\langle\int_{0} \mathrm{e}^{X_{s-}+\sigma W_{s}+\alpha t}\left(\mathrm{~d} H_{t}^{c}+\sigma \mathrm{d} W_{t}\right), H^{c}+\sigma W_{t}\right\rangle \\
& =\int_{0} \mathrm{e}^{H_{s-}+\sigma W_{t}+\alpha t} \mathrm{~d}\left\langle H_{s}^{c}+\sigma W_{s}\right\rangle \\
& =\int_{0} \mathrm{e}^{H_{s-}+\sigma W_{s}+\alpha t} \mathrm{~d} C \\
& =Y_{-} \cdot C
\end{aligned}
$$

Now

$$
\left(Y_{-} \beta\right) \cdot C=\int_{0} \beta \mathrm{e}^{H_{s-}+\sigma W_{s}+\alpha t} \mathrm{~d} C
$$

We can deduce from equating Equations (49) and (50), that Equation (40) can only hold if $\beta \equiv 1$, which is adopted as our choice of $\beta$ in this study. From the Equation (43) given by

$$
M_{\mu^{X}}^{\mathbb{P}}\left(\frac{Y}{Y_{-}} V\right)=M_{\mu^{X}}^{\mathbb{P}}
$$

And the Equation (42) which simplifies below

$$
\begin{aligned}
M_{\mu}^{\mathbb{P}}\left(\frac{Y}{Y_{-}} V\right) & =\mathbb{E}\left(\frac{Y}{Y_{-}} V \star \mu^{X}\right)_{T} \\
& =\mathbb{E}\left[\int_{0}^{T} \int_{\mathbb{R}} \frac{Y_{t}(\omega)}{Y_{t-}(\omega)} V(\omega, t, x) \mu^{X}(\omega, \mathrm{d} t, \mathrm{~d} x)\right] \\
& =\mathbb{E}\left[\int_{0}^{T} \int_{\mathbb{R}} \frac{\mathrm{e}^{H_{s}+\sigma W_{s}+\alpha t}}{\mathrm{e}^{H_{s-}+\sigma W_{s}+\alpha t}} V(\omega, t, x) \mu^{X}(\omega, \mathrm{d} t, \mathrm{~d} x)\right] \\
& =\mathbb{E}\left[\sum_{0 \leq t \leq T} \mathrm{e}^{\Delta H_{t}(\omega)} V\left(\omega, t, \Delta H_{t}(\omega) 1_{\left\{\Delta H_{t}(\omega) \neq 0\right\}}\right)\right] \\
& =\mathbb{E}\left[\int_{0}^{T} \int_{\mathbb{R}} \mathrm{e}^{\chi} V(\omega, t, x) \mu(\omega, \mathrm{d} t, \mathrm{~d} x)\right] \\
& =M_{\mu}^{\mathbb{P}}\left(\mathrm{e}^{x} V\right)
\end{aligned}
$$

We arrive at a choice of $\mathbb{Y}$ given by 


$$
\mathbb{Y}=\mathrm{e}^{x}
$$

Hence from Equation (46)

$$
B^{*}(\phi)_{t}=B(\phi)_{t}+\sigma W_{t}^{*}+H_{t}^{c}+\phi(x)\left(\mathrm{e}^{x}-1\right) \star v^{*} .
$$

Now under $\mathbb{P}^{*}$

$$
\mathcal{E}(\hat{X})_{t} \in \mathcal{M}_{\text {loc }}\left(\mathbb{P}^{*}\right) \Leftrightarrow(\hat{X})_{t} \in \mathcal{M}_{\text {loc }}\left(\mathbb{P}^{*}\right),
$$

(see [8])

From Equation (44),

$$
\mathcal{E}(X)_{t} \in \mathcal{M}_{\text {loc }}\left(\mathbb{P}^{*}\right) \Leftrightarrow G^{*}=0
$$

But in our case, to really achieve the case $G^{*}=0$, we make the following assumption: The process $1_{\{x>1\}} \mathrm{e}^{x} \star v$ has bounded variation. Provided this assumption holds and that the conditions of lemma (2.13) in [11] are satisfied, then

$$
\hat{X} \in \mathcal{M}_{\text {loc }}\left(\mathbb{P}^{*}\right) \Leftrightarrow B^{*}(\phi)_{t}+\frac{1}{2}\left(\sigma^{2} t+\left\langle H^{c}\right\rangle_{t}\right)+\left(\mathrm{e}^{x}-1-\phi(x)\right) \star v^{*}=0
$$

It is also important to take note that from the assumption we have made and lemma (2.13) in [11] above, $X_{t}$ is exponentially special i.e. $S=\mathrm{e}^{X_{t}}$ is a special semimartingale. Now

$$
\mathcal{E}(\hat{X}) \in \mathcal{M}_{\text {loc }}\left(\mathbb{P}^{*}\right) \Leftrightarrow \hat{X}_{t} \in \mathcal{M}_{\text {loc }}\left(\mathbb{P}^{*}\right)
$$

(see [12]) and from Equation (55)

$$
\mathcal{E}(\tilde{X})_{t} \in \mathcal{M}_{\text {loc }}\left(\mathbb{P}^{*}\right) \Leftrightarrow B^{*}(\phi)_{t}+\frac{1}{2}\left(\sigma^{2} t+\left\langle H^{c}\right\rangle_{t}\right)+\left(\mathrm{e}^{x}-1-\phi(x)\right) \star v^{*}=0
$$

From Equation (38)

$$
\mathrm{e}^{X_{t}} \in \mathcal{M}_{\text {loc }}\left(\mathbb{P}^{*}\right) \Leftrightarrow B^{*}(\phi)_{t}+\frac{1}{2}\left(\sigma^{2} t+\left\langle H^{c}\right\rangle_{t}\right)+\left(\mathrm{e}^{x}-1-\phi(x)\right) \star v^{*}=0
$$

This means that under $\mathbb{P}^{*}$

$$
\hat{X}_{t}=\hat{X}_{t}^{*}=H_{0}+\sigma W_{t}+H_{t}^{c}+\left(\mathrm{e}^{x}-1\right) \star(\mu-\tilde{v})
$$

Which is a martingale process.

This means that since our market has an equivalent local martingale measure $\tilde{\mathbb{P}}^{*}$ then by by the First Fundamental Theorem of Asset Pricing, there is no arbitrage in our market model ([13]).

\subsection{Equivalent Local Martingale Measures (ELMM)}

In our previous section (section 3.1), we have proved the existence of ELMM $\mathbb{P}^{*}$ and hence that the market is arbitrage free. But the question we really need to ask is whether or not $\mathbb{P}^{*}$ is a unique measure.? One of the major problems of financial markets with jumps is that they are typically incomplete. The martingale measure $\mathbb{P}^{*}$ is no longer unique as compared to complete market situation and in this case different martingale measures $\mathbb{P}^{*}$ lead to different strategies. There are more than one ELMM, in fact infinitely many. The question is that which one of them should one use in the pricing formula. To answer our question it turns out that there is a minimal entropy martingale measure $\tilde{\mathbb{P}}^{*}$ such that the optimal strategy for $\mathbb{P}$ can be computed in terms $\tilde{\mathbb{P}}^{*}$. The following discussion gives the definition of minimal martingale measure.

\section{Minimal Relative Entropy Martingale Measure (MEMM)}

Let $\mathcal{P}(S)$ be a set of all equivalent martingale measures of $Y_{t}$.

Definition $2 \tilde{\mathbb{P}}^{*}$ is said to be minimal entropy martingale measure (MEMM) of $Y_{t}$ if it satisfies the following formula 


$$
H\left(\tilde{\mathbb{P}}^{*} \mid \mathbb{P}\right) \leq H\left(\mathbb{P}^{*} \mid \mathbb{P}\right) \quad \forall \mathbb{P} \in \mathcal{P}(S)
$$

where $H\left(\mathbb{P}^{*} \mid \mathbb{P}\right)$ is the relative entropy of $\mathbb{P}^{*}$, which is given by the following formula

$$
H\left(\mathbb{P}^{*} \mid \mathbb{P}^{*}\right)= \begin{cases}\int_{\Omega} \log \frac{d \mathbb{P}^{*}}{d \mathbb{P}} \mathrm{d} \mathbb{P}^{*} & \text { if } \mathbb{P}^{*} \ll \mathbb{P} \\ +\infty & \text { otherwise }\end{cases}
$$

(see [14] [15]).

The relative entropy measures the minmal departure from a given measure $\mathbb{P}$. The relative entropy $H\left(\mathbb{P}^{*} \mid \mathbb{P}\right)$ is always non-negative and $H\left(\mathbb{P}^{*} \mid \mathbb{P}\right)_{*^{*}} 0$ is equivalent to $\mathbb{P}^{*}=\mathbb{P}$.

The minimal martingale measure $\mathbb{P}^{*}$ is uniquely determined as discussed in [14] [15].

Let $\psi=(\vartheta, \varphi, \theta) \in \mathbb{R}^{3}$ be as defined in Equation (28). We assume that the process $\vartheta=\left(\vartheta_{t}\right)_{0 \leq t \leq T}$ is predictable while $\varphi=\left(\varphi_{t}\right)_{0 \leq t \leq T}$ and $\theta=\left(\theta_{t}\right)_{0 \leq t \leq T}$ are adapted. Hence Equation (37) takes the form

$$
\mathrm{d} Y_{t}=Y_{t-} \mathrm{d} \hat{X}_{t}^{*}
$$

where $Y_{t}$ is now a martingale.

Now solving (61) above gives

$$
Y_{t}=Y_{t-} \mathcal{E}\left(\hat{X}^{*}\right)_{t}
$$

where $\mathcal{E}$ is the Dolěans Dade stochastic exponential.

$$
\mathcal{E}\left(\hat{X}^{*}\right)_{t}=\exp \left\{\hat{X}_{t}^{*}-\frac{1}{2}\left\langle\hat{X}_{t}^{*_{c}}\right\rangle_{t}\right\} \prod_{0<s \leq t}\left(1+\Delta \hat{X}_{s}^{*}\right) \mathrm{e}^{-\Delta \hat{X}_{s}^{*}} .
$$

Now

$$
\hat{X}_{t}^{*_{c}}=\sigma W_{t}+H_{t}^{c}
$$

And

$$
\begin{aligned}
\left\langle\hat{X}_{t}^{*^{c}}\right\rangle_{t} & =\left\langle\sigma \tilde{W}+H^{c}, \sigma \tilde{W}+H^{c}\right\rangle_{t} \\
& =\sigma^{2} t+\left\langle H^{c}\right\rangle_{t}
\end{aligned}
$$

(see Equation (10)). Hence

$$
\begin{aligned}
\Delta X_{t}^{*} & =\sigma \Delta W_{t}^{*}+\Delta H_{t}^{c}+\Delta\left(\mathrm{e}^{x}-1\right) \star(\mu-\tilde{v}) \\
& =\Delta H_{t}^{c}+\int\left(\mathrm{e}^{x}-1\right) \mu(\{t\} \times ; \omega)-\int\left(\mathrm{e}^{x}-1\right) v(\{t\} \times ; \omega)
\end{aligned}
$$

(for $\Delta\left(\mathrm{e}^{x}-1\right) \star(\mu-\tilde{v})$ compare with Equation (13), page 666 in [7])

Hence

$$
Y_{t}=Y_{t-} \exp \left\{X_{0}+\sigma \tilde{W}_{t}+H_{t}^{c}+\left(\mathrm{e}^{x}-1\right) \star(\mu-v)-\frac{1}{2}\left(\sigma^{2} t+\left\langle H^{c}\right\rangle_{t}\right)\right\} \prod_{0<s \leq t}\left(1+\Delta X_{t}^{*}\right) \mathrm{e}^{-\Delta X_{t}^{*}}
$$

The process $Y_{t}$, in Equation (65) above, is a martingale and it is under a new probability measure $\mathbb{P}^{*}$. This equation marks one of the major contributions of this study to the the theory option pricing through martingale approach in the sense that it accommodates both continuous and jump cases. In the situation where $\Delta H=0$, then $\mu=v=0$ and Equation (65) becomes

$$
Y_{t}=Y_{t-} \exp \left\{X_{0}+\sigma \tilde{W}_{t}+H_{t}^{c}-\frac{1}{2}\left(\sigma^{2} t+\left\langle H^{c}\right\rangle_{t}\right)\right\}
$$

which is the continuous and most familiar case, while if $\Delta H \neq 0$ then we have a jump case. The equation brings with it the convenience of converting the stock price into a martingale whenever we are using a martingale approach as in any of the cases, the process of converting the process into a martingale, simplifies to mere calculation of $\Delta H \neq 0, \mu$ and $v$ and substitute into Equation (65). 


\subsection{The Price of European Call Option}

We now come to the question fundamental of this study.

How much should the investor be willing to pay for a European call option at $t=0$ in the case where $Y_{t}$ is a semimartingale process as defined in Equation (65)?. We extend the theorem which was given in [16].

Theorem 1 Let $\hat{X}_{t}^{*}$, with its canonical decomposition as shown in Equation (59) above, be a process with independent increment, and assume that $u_{t}, \quad u_{y}$ and $u_{y y}$ exist where $u\left(t, Y_{t}\right)=\mathbb{E}^{\tilde{\mathbb{P}}^{*}}\left[\left(Y_{T}-K\right)^{+} \mid \mathcal{F}_{t}\right]$. Then the following integro-differential equation holds:

$\int_{0}^{t}\left\{u_{s}\left(s, Y_{s-}\right) \mathrm{d} s+\frac{1}{2} u_{y y}\left(s, Y_{t-}\right) Y_{s-}^{2} \mathrm{~d}\left\langle\hat{H}^{*^{*}}\right\rangle_{s}+\int_{-\infty}^{\infty}\left\{u\left(s, Y_{s-}(1+x)\right)-u\left(s, Y_{s-}\right)+u_{y}\left(s, Y_{s-}\right) Y_{s-} x\right\} \tilde{v}(\mathrm{~d} s, \mathrm{~d} x)\right\}=0$

Proof. Before we proceed, we take note of the following:

$$
\Delta Y_{t}=Y_{t-} \Delta \hat{X}_{t}^{*}, Y_{t}=Y_{t-}+\Delta Y_{t}=Y_{t-}\left(1+\Delta \hat{X}_{t}^{*}\right),\left\langle Y^{c}\right\rangle_{t}=Y_{t-}^{2}\left\langle X_{t}^{*_{c}}\right\rangle_{t}
$$

This means that $\mathrm{d} Y_{t}=Y_{t-} \mathrm{d} \hat{X}_{t}^{*}$ where

$$
\mathrm{d} \hat{X}_{t}^{*}=\mathrm{d} \hat{X}_{t}^{* c}+\int_{-\infty}^{\infty}\left(\mathrm{e}^{x}-1\right)(\mu(\mathrm{d} t, \mathrm{~d} x)-\tilde{v}(\mathrm{~d} t, \mathrm{~d} x)) .
$$

The theory of pricing of the European call option (see [17]) and the Markovian property of $Y_{t}$, the value of the European call option at time $T$ is given by

$$
V\left(T, Y_{T}\right)=\mathrm{e}^{-r T}\left[u\left(T, Y_{T}\right)\right] .
$$

where $u\left(t, Y_{t}\right)$ is given by

$$
u\left(t, Y_{t}\right)=\mathbb{E}^{\tilde{\mathbb{P}}^{*}}\left[\left(Y_{T}-K\right)^{+} \mid \mathcal{F}_{t}\right]
$$

But how do we evaluate the value $u\left(t, Y_{t}\right)$ in our case. By using the Markovian property of $Y_{t}$ we applying Ito's formula to $u\left(t, Y_{t}\right)$ and obtain

$$
\begin{aligned}
& \mathrm{d} u\left(t, Y_{t}\right) \\
= & u_{t}\left(t, Y_{t-}\right) \mathrm{d} t+u_{y}\left(t, Y_{t-}\right) \mathrm{d} Y+\frac{1}{2} u_{y y}\left(t, Y_{t-}\right) \mathrm{d}\left\langle Y^{c}\right\rangle_{t}+u\left(t, Y_{t}\right)-u\left(t, Y_{t-}\right)+u_{y}\left(t, Y_{t-}\right) \Delta Y_{t} \\
= & u_{t}\left(t, Y_{t-}\right) \mathrm{d} t+u_{y}\left(t, Y_{t-}\right) \mathrm{d} Y+\frac{1}{2} u_{y y}\left(t, Y_{t-}\right) \mathrm{d}\left\langle Y^{c}\right\rangle_{t}+u\left(t, Y_{t-}\left(1+\Delta X_{t}^{*}\right)\right)-u\left(t, Y_{t-}\right)+u_{y}\left(t, Y_{t-}\right) Y_{t-} \Delta X_{t} \\
= & u_{y}\left(t, Y_{t-}\right) \mathrm{d} Y+u_{t}\left(t, Y_{t-}\right) \mathrm{d} t+\frac{1}{2} u_{y y}\left(t, Y_{t-}\right) \mathrm{d}\left\langle Y^{c}\right\rangle_{t} \\
& +\int_{-\infty}^{\infty}\left\{u\left(t, Y_{t-}(1+x)\right)-u\left(t, Y_{t-}\right)+u_{y}\left(t, Y_{t-}\right) Y_{t-} x\right\} \tilde{v}(\mathrm{~d} t, \mathrm{~d} x) \\
u\left(t, Y_{t}\right) & \int_{0}^{t} u_{y}\left(s, Y_{t-}\right) \mathrm{d} Y \\
+ & \int_{0}^{t}\left\{u_{s}\left(s, Y_{s-}\right) \mathrm{d} s+\frac{1}{2} u_{y y}\left(s, Y_{t-}\right) Y_{s-}^{2} \mathrm{~d}\left\langle\hat{X}^{* c}\right\rangle_{s}+\int_{-\infty}^{\infty}\left\{u\left(s, Y_{s-}(1+x)\right)-u\left(s, Y_{s-}\right)+u_{y}\left(s, Y_{s-}\right) Y_{s-} x\right\} \tilde{v}(\mathrm{~d} s, \mathrm{~d} x)\right\} \\
= & \operatorname{local} \text { martingale } \\
+ & \int_{0}^{t}\left\{u_{s}\left(s, Y_{s-}\right) \mathrm{d} s+\frac{1}{2} u_{y y}\left(s, Y_{t-}\right) Y_{s-}^{2} \mathrm{~d}\left\langle\hat{X}^{* c}\right\rangle_{s}+\int_{-\infty}^{\infty}\left\{u\left(s, Y_{s-}(1+x)\right)-u\left(s, Y_{s-}\right)+u_{y}\left(s, Y_{s-}\right) Y_{s-} x\right\} \tilde{v}(\mathrm{~d} s, \mathrm{~d} x)\right\}
\end{aligned}
$$$$
=\int_{0}^{t} u_{y}\left(s, Y_{t-}\right) \mathrm{d} Y
$$

Now using the fact that a predictable local martingale with finite variation starting at zero is zero (theorem leads us to the equation i.e. 


$$
\begin{aligned}
& \mathrm{d} u\left(t, Y_{t}\right) \\
& =\int_{0}^{t}\left\{u_{s}\left(s, Y_{s-}\right) \mathrm{d} s+\frac{1}{2} u_{y y}\left(s, Y_{t-}\right) Y_{s-}^{2} \mathrm{~d}\left\langle\hat{X}^{* c}\right\rangle_{s}+\int_{-\infty}^{\infty}\left\{u\left(s, Y_{s-}(1+x)\right)-u\left(s, Y_{s-}\right)+u_{y}\left(s, Y_{s-}\right) Y_{s-} x\right\} \tilde{v}(\mathrm{~d} s, \mathrm{~d} x)\right\} \\
& =0
\end{aligned}
$$

\section{Examples}

\subsection{Example 1: Continuous Case}

Suppose $H_{t}=\delta \tilde{W}_{t}+\gamma t$

From Equation (3),

$$
X_{t}=(\alpha+\gamma) t+\sigma W_{t}+\delta \tilde{W}_{t}
$$

where $\tilde{W}_{t}$ is the Brownian Motion on the same stochastic basis $(\Omega, \mathcal{F}, \mathbb{F}, \mathbb{P})$ as Brownian Motion $W_{t}$ and that these Brownian Motions are correlated with correlation co-efficient $\rho$. Obviously, from Equation (68), $\Delta X_{t}=0$.

$$
\mu_{k}(U ; \omega)=I\left(\Delta X_{k}(\omega) \in U, U \in \mathcal{B}\left(\mathbb{R}^{d} \backslash\{0\}\right)=0 .\right.
$$

Note that $U$, as it is defined in Equation (69), is an element of Borel sets which do not have a 0 element. Since $\Delta X_{t}=0$ and 0 cannot be an element of $U$ justifies Equation (69).

It follows that

$$
\mu_{(0, n]}^{X}(U ; \omega)=\sum_{k=1}^{n} \mu_{k}^{X}(U ; \omega)=0
$$

Similarly

$$
v_{n}(U ; \omega)=\mathbb{P}\left(\Delta X_{n} \in U \mid \mathbb{F}_{n-1}(\omega)\right)(\mathbb{P}-\text { a.s })=0
$$

From Equations (39) and (51)), under measure $\mathbb{P}^{*}$,

$$
v^{*}=v \cdot \mathbb{Y}=0 \cdot \mathrm{e}^{x}=0
$$

And $\mu-\tilde{v}=0$. Hence from Equation (64)

$$
\begin{aligned}
\Delta \hat{X}_{t}^{*} & =\int\left(\mathrm{e}^{x}-1\right) \mu(\{t\} \times ; \omega)-\int\left(\mathrm{e}^{x}-1\right) v(\{t\} \times ; \omega) \\
& =0
\end{aligned}
$$

Hence $\hat{X}_{t}^{*_{c}}=\sigma W_{t}^{*}+\delta \tilde{W}_{t}^{*},\left\langle\hat{X}^{*_{c}}\right\rangle_{t}=\left(\sigma^{2}+\delta^{2}\right) t \quad$ and $\mathrm{d}\left\langle\hat{X}^{*_{c}}\right\rangle=\left(\sigma^{2}+\delta^{2}\right) \mathrm{d} t$. Using theorem (1) with $x=0$ and $\tilde{v}=0$ leads to equation

$$
u_{t}\left(t, Y_{t-}\right) \mathrm{d} t+\frac{1}{2}\left(\sigma^{2}+\sigma_{*}^{2}\right) Y_{t-}^{2} u_{y y}\left(t, Y_{t-}\right)=0
$$

And hence (from the same theorem)

$$
V\left(T, Y_{T}\right)=\mathrm{e}^{-r T} u\left(T, Y_{T}\right)
$$

\subsection{Example 2: Process with Jump}

Suppose in our model, the exchange rate is not continuous and is modeled by the stochastic differential equation

$$
\mathrm{d} E_{t}=\left[\alpha_{*} E_{-} \mathrm{d} t+\sigma_{*} E_{t-} \mathrm{d} W_{*_{t}}+\left(\mathrm{e}^{\varphi}-1\right) E_{t-} \mathrm{d} M_{t}\right]
$$

where $W_{*_{t}}$ is the $\mathbb{P}$-Brownian Motion process, $M_{t}$ is the compensated $\mathbb{P}$-martingale process associated with the Poisson process $N_{t}$ (with intensity $\varrho$ ) i.e. $M_{t}=N_{t}-\varrho t$ and is independent of the Brownian motion $N_{t}$. We choose $\varphi_{t}$ to be a Gaussian process with independent increments, independent to both $W_{*_{t}}$ and $N_{t}$. We let $\mathbb{E}\left(\varphi_{t}\right)=\eta$ and $\mathbb{V}\left(\varphi_{t}\right)=\tau^{2}$. Assume that $\sigma_{*}>0, \tau>0, \eta \in \mathbb{R}$. We further assume that $W_{*_{t}}$ is independent of $W_{t}$ where $W_{t}$ is the Brownian motion as defined in Equation (3). 
To find out what our $X_{t}$ and $\hat{H}_{t}^{*}$, are in this case we first of all solve our equation.

$$
\frac{\mathrm{d} E_{t}}{E_{t-}}=\left(\alpha_{*}+\frac{1}{2} \sigma_{*}\right) \mathrm{d} t+\sigma_{*} W_{* t}+\left(\mathrm{e}^{\varphi}-1\right) \mathrm{d} M_{t} .
$$

Using Itó's formula for processes with jumps, we let

$$
\begin{aligned}
& Y_{t}=\log \left(E_{t}\right) \\
& \mathrm{d}\left(\log E_{t}\right)= \frac{1}{E_{t_{-}}} \mathrm{d} E_{t}+\frac{1}{2}\left(-\frac{1}{E_{t_{-}}^{2}}\right) \sigma_{*}^{2} E_{t_{-}}^{2} \mathrm{~d} t+\left(\log E_{t}-\log E_{t-}-\frac{1}{E_{t_{-}}}\left(\mathrm{e}^{\varphi}-1\right) E_{t-}\right) \mathrm{d} N_{t} \\
&= \frac{1}{E_{t_{-}}}\left(\alpha_{*} E_{t_{-}} \mathrm{d} t+\sigma_{*} E_{t_{-}} \mathrm{d} W_{*_{t}}-\left(\mathrm{e}^{\delta}-1\right) E_{t_{-}} \mathrm{d} M_{t}\right)+\frac{1}{2}\left(-\frac{1}{E_{t_{-}}^{2}}\right) \sigma_{*}^{2} E_{t_{-}}^{2} \mathrm{~d} t+\left(\log \left[\mathrm{e}^{\phi} E_{t_{-}}\right]-\log E_{t_{-}}\right) \mathrm{d} N_{t} \\
&=\left(\alpha_{*}-\frac{1}{2} \sigma_{*}\right) \mathrm{d} t+\sigma_{*} \mathrm{~d} W_{*_{t}}+\left(\mathrm{e}^{\varphi}-1\right)\left(\mathrm{d} N_{t}-\lambda \mathrm{d} t\right)+\log \frac{\mathrm{e}^{\varphi} E_{t_{-}}}{E_{t_{-}}} \mathrm{d} N_{t}-\left(\mathrm{e}^{\varphi}-1\right) \mathrm{d} N_{t} \\
&=\left(\alpha_{*}-\frac{1}{2} \sigma_{*}\right) \mathrm{d} t+\sigma_{*} \mathrm{~d} W_{*_{t}}+\left(\mathrm{e}^{\varphi}-1\right) \mathrm{d} N_{t}-\lambda\left(\mathrm{e}^{\varphi}-1\right) \mathrm{d} t+\varphi \mathrm{d} N_{t}-\left(\mathrm{e}^{\varphi}-1\right) \mathrm{d} N_{t} \\
&=\left(\alpha_{*}-\frac{1}{2} \sigma_{*}-\lambda\left(\mathrm{e}^{\varphi}-1\right)\right) \mathrm{d} t+\sigma_{*} \mathrm{~d} W_{*_{t}}+\varphi \mathrm{d} N_{t}
\end{aligned}
$$

From which we obtain

$$
\log E_{t}=\left(\alpha_{*}-\frac{1}{2} \sigma_{*}^{2}-\lambda\left(\mathrm{e}^{\varphi}-1\right)\right) t+\sigma W_{* t}+\varphi N_{t}
$$

And

$$
E_{t}=E_{0} \exp \left\{\alpha_{*}-\frac{1}{2} \sigma^{2}-\lambda\left(\mathrm{e}^{\varphi}-1\right) t+\sigma W_{*_{t}}+\varphi N_{t}\right\} .
$$

Hence from Equation (77), we obtain $H_{t}$ given by

$$
H_{t}=\alpha_{*}-\frac{1}{2} \sigma^{2}-\lambda\left(\mathrm{e}^{\varphi}-1\right) t+\sigma W_{* t}+\varphi N_{t} .
$$

Clearly, $H_{0}=0$ (since $W_{0}=N_{0}=0$ ). From Equation (2), we obtain

$$
\begin{aligned}
Y_{t} & =E_{0} S_{0} \exp \left\{\alpha t+\sigma W_{t}+H_{t}\right\} \\
& =Y_{0} \exp \left\{\left(\alpha+\alpha_{*}-\frac{1}{2} \sigma^{2}-\lambda\left(\mathrm{e}^{\varphi}-1\right)\right) t+\sigma W_{t}+\sigma_{*} W_{*_{t}}+\varphi N_{t}\right\}
\end{aligned}
$$

From (78) we obtain the process of the form

$$
X_{t}=\left(\alpha+\alpha_{*}-\frac{1}{2} \sigma^{2}-\frac{1}{2} \sigma_{*}^{2}-\lambda\left(\mathrm{e}^{\varphi}-1\right)\right) t+\sigma W_{t}+\sigma_{*} W_{* t}+\varphi N_{t}
$$

Equation (79) yields the sharp bracket process for $X_{t}$ of the form

$$
X_{t}^{c}=\sigma W_{t}+\sigma_{*} W_{*_{t}}
$$

And hence under measure $\tilde{\mathbb{P}}^{*}$,

$$
\hat{X}_{t}^{*}=\alpha t+\sigma \tilde{W}_{t}+\sigma_{*} \tilde{W}_{*_{t}}+\int_{0}^{t} \int_{-\infty}^{\infty}\left(\mathrm{e}^{x}-1\right)(\mu(\mathrm{d} s, \mathrm{~d} x)-v(\mathrm{~d} s, \mathrm{~d} x))
$$

Clearly $\hat{X}_{t}^{*}(80)$ is a process with independent increments (as it is the sum of processes with independent increments), hence we can find a deterministic function $c_{t}$, a deterministic measure-valued function $\bar{K}_{t}$ and a deterministic increasing function $L_{t}$ such that $\left.\mathrm{d}\left\langle X^{c}\right\rangle_{t}\right\rangle_{t}(\omega)=c_{t} \mathrm{~d} L_{t}(\omega), \quad v(\mathrm{~d} t, \mathrm{~d} x)=\bar{K}_{t}(\mathrm{~d} x) \mathrm{d} L_{t}(\omega){ }^{\prime}(\operatorname{Jacod}$ 
and Shiryaev [8]). $\hat{X}_{t}^{*}$ is also a Lèvy process, we can choose $L_{t}=t, c$ to be a constant and $\bar{K}(\mathrm{~d} x)$ (the Levy measure) (see section). In addition, $\bar{K}(\mathrm{~d} x)=\varrho \cdot F(\mathrm{~d} x)$ where $\varrho=\mathbb{E}\left(N_{t}\right)$ and $F(\mathrm{~d} x)$ is the distribution of the jump size of less than 1, i.e. from this we can deduce that the expected number of jumps, in time interval 1 is $\varrho$ and the jump size is distributed according to $F$. If we let $\mathrm{e}^{\varphi_{t}}-1=x$ then $\varphi_{t}=\ln (x+1)$. Since $\varphi_{t}$ is a Gaussian process with $\mathbb{E}\left(\varphi_{t}\right)=\eta$ and $V\left(\varphi_{t}\right)=\tau^{2}$, Then

$$
F(\mathrm{~d} x)=\frac{1}{\sqrt{2 \pi \tau}} \cdot \exp \left\{-\frac{(\ln (x+1)-\eta)^{2}}{\tau^{2}}\right\}
$$

And

$$
\begin{aligned}
\bar{K}(x) & =\varrho \cdot F(\mathrm{~d} x) \\
& =\frac{\varrho}{\sqrt{2 \pi \tau}} \cdot \exp \left\{-\frac{(\ln (x+1)-\eta)^{2}}{\tau^{2}}\right\}
\end{aligned}
$$

From Equation (80),

$$
\left\langle\hat{X}_{t}^{*}\right\rangle=\left\langle\sigma W_{t}+\sigma_{* t} W_{*_{t}}\right\rangle=\left(\sigma+\sigma_{*}\right) t
$$

And

$$
\mathrm{d}\left\langle\hat{X}^{*}\right\rangle_{t}=\left(\sigma+\sigma_{*}\right) \mathrm{d} t
$$

Hence from theorem (1), $u\left(t, Y_{t}\right)$ is found by solving the equation

$$
\begin{aligned}
& u_{t}\left(t, Y_{t-}\right) \mathrm{d} t+\frac{1}{2}\left(\sigma^{2}+\sigma_{*}^{2}\right) Y_{t-}^{2} u_{y y}\left(t, Y_{t-}\right) \\
& +\left\{u\left(t, Y_{t-}(1+\varphi)\right)-u\left(t, Y_{t-}\right)+u_{y}\left(t, Y_{t-}\right) Y_{t-} \varphi\right\} \varrho \varphi=0
\end{aligned}
$$

And

$$
V\left(T, Y_{T}\right)=\mathrm{e}^{-r T} u\left(T, Y_{T}\right)
$$

\subsection{Example 3: $H_{t}$ a Cumulative Process}

We consider a situation where the exchange rate is $E=E_{0} \exp \{h \circ Z\}$ where $W_{t}$ and $Z_{t}$ are independent processes. In this case $H_{t}=h \circ Z$. This means that

$$
X_{t}=\alpha t+\sigma W_{t}+H_{t}
$$

In this case

$$
\hat{X}^{\star c}=\sigma W_{t}^{\star}+h \circ Z^{c}
$$

This means that

$$
\begin{aligned}
\left\langle\hat{X}^{\star c}\right\rangle & =\left\langle\sigma W^{\star}+h \circ Z^{c}\right\rangle_{t} \\
& =\left\langle\sigma W^{\star}\right\rangle_{t}+2\left\langle\sigma W, h \circ Z^{c}\right\rangle_{t}+\left\langle h \circ Z^{c}, h \circ Z^{c}\right\rangle_{t} \\
& =\sigma^{2} t+2 \sigma\left\langle W, h \circ Z^{c}\right\rangle_{t}+h^{2} \circ\left\langle Z^{c}\right\rangle_{t} \\
& =\sigma^{2} t+h^{2} \circ\left\langle Z^{c}\right\rangle_{t}
\end{aligned}
$$

Hence Equation (80) becomes

$$
\hat{X}_{t}^{*}=\alpha t+\sigma \tilde{W}_{t}+h \circ Z^{c}+\int_{0}^{t} \int_{-\infty}^{\infty}\left(\mathrm{e}^{x}-1\right)(\mu(\mathrm{d} s, \mathrm{~d} x)-v(\mathrm{~d} s, \mathrm{~d} x))
$$

This means $u\left(t, Y_{t}\right)$ is the solution of the integro-differential equation 


$$
\begin{aligned}
& \int_{0}^{t}\left\{u_{s}\left(s, Y_{s-}\right) \mathrm{d} s+\frac{1}{2} u_{y y}\left(s, Y_{t-}\right) Y_{s-}^{2} \mathrm{~d}\left(\sigma^{2} t+h^{2} \circ\left\langle Z^{c}\right\rangle_{t}\right)\right. \\
& \left.\quad+\int_{-\infty}^{\infty}\left\{u\left(s, Y_{s-}(1+x)\right)-u\left(s, Y_{s-}\right)+u_{y}\left(s, Y_{s-}\right) Y_{s-} x\right\} \tilde{v}(\mathrm{~d} s, \mathrm{~d} x)\right\}=0
\end{aligned}
$$

With

$$
V\left(T, Y_{T}\right)=\mathrm{e}^{-r T} u\left(T, Y_{T}\right)
$$

\section{Discussion}

Equation (73) compares well with Equation (82) in the sense that (82) without the term

$$
\left\{u\left(t, Y_{t-}(1+\varphi)\right)-u\left(t, Y_{t-}\right)+u_{y}\left(t, Y_{t-}\right) Y_{t-} \varphi\right\} \varrho \varphi
$$

Gives Equation (73). This means that Equation (85) is the contribution of the jump to the price of the option. The effects of the jumps on the price of the the option can be easily observed from this Equation (82) through the role $\varphi$, which is the jump parameter in this equation. For example in the case where $\varphi=0$, we

$$
\left\{u\left(t, Y_{t-}(1+\varphi)\right)-u\left(t, Y_{t-}\right)+u_{y}\left(t, Y_{t-}\right) Y_{t-} \varphi\right\} \varrho \varphi=0
$$

Hence Equation (82) is reduced to Equation (73) which is a continuous case. This can be further justified from the definition of our $\varphi . \varphi=\ln (1+x)$ and hence $\varphi=0 \Leftrightarrow x=0$ i.e. $\varphi=0$ if $\Delta X=0$, justifying why case (2) in section (4.2) is reduced to case (1) in section (4.1) and at the same time confirming why Equation (82) degenerates to equation (73). We also take note that the co-domain of the function $x \rightarrow \varphi(x)$ is $[0, \infty)$. This means $\varphi$ is not taking negative values hence has a positive effect to the value of the option. The positive effect of $\varphi$ is in the sense that as far as

$$
\left\{u\left(t, Y_{t-}(1+\varphi)\right)-u\left(t, Y_{t-}\right)+u_{y}\left(t, Y_{t-}\right) Y_{t-} \varphi\right\} \varrho>0
$$

In Equation (82), the increase in $\varphi$ will mean the increase in the price of the option In the same vein, $\varrho$ depends on $\varphi$ as $\mathbb{E}(\varphi)=\varrho$ hence we expect $\varrho>0$ as $\varphi$ takes positive values. This means $\varrho$ has a positive effect in Equation (82), emphasizing the effects of $\varphi$.

We also take note that expression (85) is also equal to zero if either $\varphi=0$ (the case which we have just discussed), $\varrho=0$ (the same case since $\varrho=0$ (in our case) if and only if $\varphi=0$ ) or if

$$
u\left(t, Y_{t-}(1+\varphi)\right)-u\left(t, Y_{t-}\right)+u_{y}\left(t, Y_{t-}\right) Y_{t-} \varphi=0
$$

A case which can be handled numerically.

\section{Conclusion}

The method gives the general method of calculating the price of the option in the sense that it accommodates both continuous and processes with jumps. When $\Delta X_{t}=0$, we are talking of continuous processes as shown by example 1 and when $\Delta X_{t} \neq 0$ we are talking of processes with jumps. This can provide a more general way of finding the price of an option.

\section{References}

[1] Akigirayi, V. and Booth, G. (1988) Mixed Diffusion-Jump Process Modeling of Exchange Rate Movements. Review of Economics and Statistics, 70, 631-637. http://www.jstor.org/stable/1935826

[2] Etheridge, A. (2002) A Course in Financial Calculus. Cambridge Press, Cambridge. http://dx.doi.org/10.1017/CBO9780511810107

[3] Jorion, P. (1988) On Jump Processes in Foreign Exchange and Stock Markets. Review of Financial Studies, 1, $427-445$. http://dx.doi.org/10.1093/rfs/1.4.427

[4] Merton, R. (1976) Option Pricing When Underlying Stock Returns Are Discontinuous. Journal of Financial Economics, 3, 125-144. http://dx.doi.org/10.1016/0304-405X(76)90022-2 
[5] Press, J.A. (1967) A Compound Events Model of Security Prices. Journal of Business, 40, 317-335. http://dx.doi.org/10.1086/294980

[6] Protter, P. (1992) Stochastic Integration and Differential Equations: A New Approach. Springer-Verlag, Berlin.

[7] Shiryaev, A.N. (1999) Essentials of Stochastic Finance, Facts, Models, Theory. World Scientific Pub Co Inc., Hackensack.

[8] Jacod, J. and Shiryaev A.N. (1987) Limit Theorems for Stochastic Processes. Springer, Berlin. http://dx.doi.org/10.1007/978-3-662-02514-7

[9] Klebanner, F. (2005) Introduction to Stochastic Calculus with Applications. Imperial College Press, Berlin. http://dx.doi.org/10.1142/p386

[10] Papapantoleoen, A. (2006) Application for Semimartingales and Lèvy Processes in Finance: Duality and Valuation. Dissertation zur Erlangung des Doktorgrades der Fakultät für Mathematik und Physik der Albert-Ludwigs-Universitat, Freiburg im Breisgau.

[11] Kallesen, J. and Shiryaev, A. (2002) The Cummlant Process and Esscher's Change of Measure. Finance and Stochastics, 6, 397-428. http://dx.doi.org/10.1007/s007800200069

[12] Bulhman, H., Delbaen, F., Embrechts, P. and Shiryaev, A.N. (1996) No-Arbitrage, Change of Measure and Conditional Esscher's Transforms. CW Quartery, 9, 291-317.

[13] Harrison, J. and Pliska, S. (1981) Martingales and Stochastic Integrals in Theory of Continuous Trading. Stochastic Processes and Their Applications, 11, 215-260. http://dx.doi.org/10.1016/0304-4149(81)90026-0

[14] Follmer, H. and Schwaizer, M. (1991) Hedging Contingent Claims under Incomplete Information. In: Davis, M.A. and Elliot, R.J., Eds., Applied Stochastic Analysis Monographs, 4th Edition, Vol. 5, Gordon and Breach, London, 389-414.

[15] Miyahara, Y. (1999) Minimal Entropy Martingale Measures Jump Type Price Process in Incomplete Asset Markets. Asian-Pacific Financial Markets, 6, 97-113.

[16] Vecer, J. (2003) Pricing Asian Options in Semimartingale Model. Columbia University, New York.

[17] Baxter, M. and Rennie, A. (1996) Financial Calculus: An Introduction to Derivative Pricing. Cambridge University Press, Cambridge, England. http://dx.doi.org/10.1017/CBO9780511806636

\section{Appendix}

Then, using Ito's formula for semimartingales (Protter [6]), we have

$$
\begin{aligned}
Y_{t} & =\mathrm{e}^{X_{0}}+\int_{0}^{t}\left(\mathrm{e}^{X_{s-}}\right)^{\prime} \mathrm{d} X_{s}+\frac{1}{2} \int_{0}^{t}\left(\mathrm{e}^{X_{s-}}\right)^{\prime \prime} \mathrm{d}\left\langle X^{c}\right\rangle_{s}+\sum_{0 \leq s \leq t}\left(\mathrm{e}^{X_{s}}-\mathrm{e}^{X_{s-}}-\left(\mathrm{e}^{X_{s-}}\right)^{\prime} \Delta X_{s}\right) \\
& =\mathrm{e}^{X_{0}}+\int_{0}^{t} \mathrm{e}^{X_{s-}} \mathrm{d} X_{s}+\frac{1}{2} \int_{0}^{t} \mathrm{e}^{X_{s-}} \mathrm{d}\left\langle X^{c}\right\rangle_{s}+\sum_{0 \leq s \leq t}\left(\mathrm{e}^{X_{s}}-\mathrm{e}^{X_{s-}}-\mathrm{e}^{X_{s-}} \Delta X_{s}\right)
\end{aligned}
$$

And in differential form, this can be expressed as

$$
\begin{aligned}
\mathrm{d} Y_{t} & =\mathrm{e}^{X_{t-}} \mathrm{d} X_{t}+\frac{1}{2} \mathrm{e}^{X_{t-}} \mathrm{d}\left\langle X^{c}\right\rangle_{t}+\mathrm{e}^{X_{t}}-\mathrm{e}^{X_{t-}}-\mathrm{e}^{X_{t-}} \Delta X_{t} \\
& =\mathrm{e}^{X_{t-}} \mathrm{d} c X_{t}+\frac{1}{2} \mathrm{e}^{X_{t-}} \mathrm{d}\left\langle X^{c}\right\rangle_{t}+\mathrm{e}^{X_{t-}+\Delta X_{t}}-\mathrm{e}^{X_{t-}}-\mathrm{e}^{X_{t-}} \Delta X_{t} \\
& =\mathrm{e}^{X_{t-}} \mathrm{d} X_{t}+\frac{1}{2} \mathrm{e}^{X_{t-}} \mathrm{d}\left\langle X^{c}\right\rangle_{t}+\mathrm{e}^{X_{t-}}\left(\mathrm{e}^{\Delta X_{t}}-1-\Delta X_{t}\right) \\
& =\mathrm{e}^{X_{t-}} \mathrm{d}\left[X_{t}+\frac{1}{2}\left\langle X^{c}\right\rangle_{t}+\sum_{0<s \leq t}\left(\mathrm{e}^{\Delta X_{s}}-1-\Delta X_{s}\right)\right] \\
& =Y_{t-} \mathrm{d} \hat{X}_{t},
\end{aligned}
$$

